\title{
Article \\ Identification and Characterization of the Detoxification Genes Based on the Transcriptome of Tomicus yunnanensis
}

\author{
Wen $\mathrm{Li}^{1}$, Bin Yang ${ }^{2}$, Naiyong Liu ${ }^{2}$, Jiaying Zhu ${ }^{2}{ }^{-}$, Zongbo $\mathrm{Li}^{2}$, Sangzi Ze ${ }^{3}$, Jinde $\mathrm{Yu}^{1}$ and Ning Zhao ${ }^{1,2, *}$ \\ 1 College of Life Sciences, Southwest Forestry University, Kunming 650224, China; liwenaya@163.com (W.L.); \\ yujinde@swfu.edu.cn (J.Y.) \\ 2 Key Laboratory of Forest Disaster Warning and Control of Yunnan Province, Southwest Forestry University, \\ Kunming 650224, China; yangbin48053@swfu.edu.cn (B.Y.); naiyong.liu@swfu.edu.cn (N.L.); \\ jyzhu@swfu.edu.cn (J.Z.); lizb@swfu.edu.cn (Z.L.) \\ 3 Yunnan Forestry and Grassland Pest Control and Quarantine Bureau, Kunming 650051, China; \\ zesangzi@163.com \\ * Correspondence: lijiangzhn@swfu.edu.cn
}

check for updates

Citation: Li, W.; Yang, B.; Liu, N.; Zhu, J.; Li, Z.; Ze, S.; Yu, J.; Zhao, N. Identification and Characterization of the Detoxification Genes Based on the Transcriptome of Tomicus yunnanensis. Diversity 2022, 14, 23 https://doi.org/10.3390/d14010023

Academic Editor: Luc Legal

Received: 27 November 2021

Accepted: 29 December 2021

Published: 31 December 2021

Publisher's Note: MDPI stays neutral with regard to jurisdictional claims in published maps and institutional affiliations.

Copyright: (c) 2021 by the authors. Licensee MDPI, Basel, Switzerland. This article is an open access article distributed under the terms and conditions of the Creative Commons Attribution (CC BY) license (https:// creativecommons.org/licenses/by/ $4.0 /)$.
Abstract: Bark beetle, as a trunk borer, has caused a large number of tree deaths and seriously damaged the mountain forest ecosystem. Bark beetles oxidize the secondary metabolites of plants, degrade them, and excrete them from the body or convert them into components needed by the body. This process is completed by the cooperation of CYPs, GSTs, and CCEs and occurs in different tissues of the insects, including the gut (i.e., the part where beetle pheromone is produced and accumulated) and antennae (i.e., the olfactory organ used to sense defensive monoterpenes and other plant-related compounds and pheromones in the air). In this study, we identified and characterized three gene superfamilies of CYPs, GSTs, and CCEs involved in the detoxification of endobiotics (e.g., hormones and steroids) and xenobiotics (e.g., insecticides, sex pheromones, and plant allelochemicals) through a combination approach of bioinformatics, phylogenetics, and expression profiles. Transcriptome analyses led to the identification of 113 transcripts encoding 51 P450s, 33 GSTs, and 29 CCEs from Tomicus yunnanensis Kirkendall and Faccoli, 2008 (Coleoptera, Scolytinae). The P450s of T. yunnanensis were phylogenetically classified into four clades, representing the majority of the genes in the CYP3 clan. The CCEs from T. yunnanensis were separately grouped into five clades, and the GST superfamily was assigned to five clades. Expression profiles revealed that the detoxification genes were broadly expressed in various tissues as an implication of functional diversities. Our current study has complemented the resources for the detoxification genes in the family Coleoptera and allows for functional experiments to identify candidate molecular targets involved in degrading plants' secondary metabolites, providing a theoretical basis for insect resistance in mixed forests.

Keywords: cytochrome P450; glutathione S-transferase; carboxylesterases; bark beetles; gene expression

\section{Introduction}

To withstand the ingestion of insects, plants have developed a defense mechanism against insects through various methods such as morphology, biochemistry, and molecular regulation in the long-term evolution process [1]. The production of secondary metabolites in plants is a way of defending themselves against insects. Various secondary metabolites in plants can affect the feeding and food utilization of insects, and toxic secondary metabolites can lead directly to insect death or stunted growth [2,3].

However, in the long-term evolutionary process, insects are constantly adapting to plant defense mechanisms. Genetic variation in morphology, including polymorphic wing growth, and differentiation of different types of mouthparts, such as chewing, stabbing, and sucking [4]. Insects can also use the enzymatic detoxification system, which mainly includes cytochrome P450 monooxygenases (CYPs), glutathione S-transferases (GSTs), and 
carboxylesterases (CCEs), to improve the ability of insects to metabolize secondary substances of plants, and cause insects to adapt to the defense mechanism of the host plant [5].

CYPs are a very large and diverse group of enzymes found in all living things. They constitute a very important system involving endogenous compounds and xenobiotics such as drugs, pesticides, secondary plant metabolites, mutagens, and hormones [6,7]. In addition, CYPs can reflect different levels of phylogenetic information such as subtypes, population, and species. It is a good molecular marker to explore the phylogenetic relationship between populations and population genetic diversity $[8,9]$. According to the similarity of amino acids, the CYPs of insects can be divided into four clades: CYP2, CYP3, CYP4, and mitochondrial CYP clades [10,11]. When exposed to toxic plant compounds, insects will induce reactions to other exogenous substances. For example, CYP6AE14 in Helicoverpa armigera Hübner, 1809 (Lepidoptera, Noctuidae) is involved in the metabolism of gossypol (plant toxins) [12]. GST is a dimeric protein, which plays an important role in intracellular metabolism, chemical cycle, and body defense [13]. Insect GST can be divided into six categories (delta, epsilon, omega, sigma, theta, and zeta). Among them, the delta and epsilon subtypes are unique to insects, and some GSTs play an important role in plant antitoxin [14,15]. In research on Dendroctonus armandi Tsai and Li, 1959 (Coleoptera, Scolytinae), it is assumed that DaGSTe1 catalyzes the combination of glutathione with terpenes and phenolic substances from $D$. armandi, so that toxic substances are transported out of the cell, thereby reducing the damage of exogenous toxins from the host to D. armandi [16]. In addition to CYPs and GSTs, another detoxification enzyme is CCE. Insect CCEs belong to the carboxyl/cholinesterase family, a branch of the $\alpha / \beta$-hydrolase fold superfamily in which enzymes hydrolyze ester bonds, which are present in many plant volatiles, insect pheromones, and hormones, as well as pesticides [17].

Tomicus yunnanensis Kirkendall and Faccoli, 2008 (Coleoptera, Scolytinae) is one of the main pests of Pinus yunnanensis, which is widely distributed in Yunnan, Sichuan, and Guizhou. The whole life cycle of T. yunnanensis is almost entirely on P. yunnanensis [18]. The damage to P. yunnanensis by T. yunnanensis can be divided into two periods: feeding on treetops and trunks. When feeding on the treetops, the adult worms feed on the pith tissues of the branches and supplement nutrients to complete their sexual development; when feeding on the trunk, the mature adult worms transfer from the treetop to the trunk and feed on phloem, lay eggs, and form mother tunnel and sub tunnel. Then, larvae develop under the bark, pupate, and emerge, and adults will burrow out of the phloem and return to the treetops to feed $[19,20]$. This concealed lifestyle brings many inconveniences to the study of its habits, prevention, and treatment. T. yunnanensis first broke out in central Yunnan Province in the 1980s, and then spread to 65 counties in 15 regions of Yunnan Province, infesting more than 200,000 ha of Yunnan pine forests and blighting more than 93,000 ha of P. yunnanensis, and seriously damaged the mountain forest ecosystem [21]. To mitigate the damage of this insect, some common measures can play a certain role for a short time, such as biological control, chemical control and tending, and mixed forest [22]. Among them, the mixed forest has achieved remarkable results in insect resistance and contributed to the protection of mountain forest ecosystem, but its anti-insect mechanism still needs further research. The identification of the detoxification genes of T. yunnanensis enriches the detoxification gene family in Coleoptera and provides data support for future research on the function of detoxification genes. We hope that the study can provide a theoretical basis for insect resistance in mixed forests.

\section{Materials and Methods}

\subsection{Insect and Tissue Collection}

The adults of T. yunnanensis used in this experiment were originally collected from Jiulong Mountain Forest Farm in Zhanyi County, Qujing City, Yunnan Province in June 2018. The antennae, heads, legs, and carcasses (excluding antennae, heads, and legs) of 200 pairs of male and female adults of T. yunnanensis were collected separately (three groups of 
biological replicates). We quickly froze the collected samples with liquid nitrogen and stored them in a refrigerator at $-80^{\circ} \mathrm{C}$. qPCR samples were treated the same way.

\subsection{Total RNA Extraction and $c D N A$ Synthesis}

Total RNA samples of tissues were isolated using TRIzol Reagent according to the manufacturer's protocol (Ambion, Life Technologies, Carlsbad, CA, USA). The quality of RNA was confirmed using a NanoVue UV-vis spectrophotometer (Thermo Fisher Scientific, Waltham, MA, USA), and RNA integrity was verified using a standard $1 \%$ agarose gel electrophoresis. Genomic DNA was digested by treatment with DNase I (Fermentas, Thermo Fisher Scientific, USA). First-strand cDNA was synthesized with a first strand cDNA synthesis kit (TaKaRa, Dalian, Liaoning, China). The synthesized cDNA templates were stored at $-20^{\circ} \mathrm{C}$.

\subsection{Library Construction, Sequencing, and Functional Annotation}

Oligosaccharide (DT)-containing magnetic beads were used to enrich the mRNAs in the total RNA of antennae, head, foot, and residue. Then, according to the protocol in NEBNext ${ }^{\circledR}$ Ultra $^{\mathrm{TM}}$ RNA Library Prep Kit for llumina ${ }^{\circledR}$ (NEB, Ipswich, MA, USA), we cut them into short fragments with a fragment buffer. The first-strand cDNA was synthesized using this fragment as a template, and then the second-strand cDNA was synthesized by DNA polymerase I and RNaseH. After purification with AMPure XP beads, the second-strand cDNA was repaired, $\alpha$-end ligated, and ligated with indexed adapters. The products of suitable size were selected, amplified by PCR, and purified with AMPure $\mathrm{XP}$ beads to establish a digital gene expression (DEG) library. Raw reads were processed through a rigorous filtering process to eliminate low-quality reads (base calling). The clean reads were then assembled using Trinity (v2.4.0) and then grouped using Corset (v1.0.5) to eliminate redundant data in the assembled transcripts. [23]. Databases used for annotation included the non-redundant nucleotide $(\mathrm{Nt})$, non-redundant database $(\mathrm{Nr})$, Swiss-Prot, Protein family (Pfam), Kyoto Encyclopedia of Genes and Genomes (KEGG) and Clusters of Orthologous Groups (COG/KOG). Gene ontology (GO) analysis was performed using blast2go (b2g4pipe_v2.5) software. Nr, Swiss-Prot, and COG/KOG were analyzed by diamond v0.8.22 software, and Nt was analyzed by NCBI blast v2.2.28+; KEGG was analyzed with KAAS r140224 and Pfam was analyzed with hmmscan HMMER 3. For the DEG analysis, the resulting clean reads were then mapped to the transcriptomic unigenes using RSEM software with default parameters [24]. The differential expression of different conditions/groups (genes and samples) was analyzed by DESeq2 software. The number of read counts normalized by TMM for each mapped gene was used to calculate gene expression levels following the FPKM (fragments per kilobase of transcript sequence per millions base) method $[25,26]$.

\subsection{Gene Identification and Sequence Analysis}

To identify candidate detoxification genes from T. yunnanensis, detoxification gene families from other coleopteran species were selected as queries to search the new stand-alone transcriptome of this beetle. Anoplophora glabripennis Motschulsky, 1854 (Coleoptera, Lamiinae), Lepeophtheirus salmonis Kroyer, 1838 (Copepoda, Caligoida), Dendroctonus ponderosne Hopkins, 1902 (Coleoptera, Scolytidae), and Tribolium castaneum Herbst, 1797 (Coleoptera, Tenebrionidae) were used for CYPs; D. ponderosae, A. glabripennis, Rhynchophorus ferrugineus Oliver, 1790 (Coleoptera: Curculionidae), D. armandi and T. castaneum for GSTs, and D. ponderosae, D. armandi, and T. castaneum for CCEs (https:/ / www.ncbi.nlm.nih.gov / (accessed on 7 June 2021)). TBLASTN was used to search and identify candidate detoxification genes against the T. yunnanensis transcriptome, with an E-value cutoff of $1 \times 10^{-5}$. Further, these identified genes were verified using TBLASTX against the NCBI non-redundant protein sequences database. Open reading frames (ORFs) were identified using the ORF Finder in NCBI (https: / / www.ncbi.nlm.nih.gov / orffinder/ (accessed on 29 June 2021)). CYP names use the CYP prefix, followed by an Arabic numeral, designates the family (all 
members nominally $>40 \%$ identical), a capital letter designates the subfamily (all members nominally $>55 \%$ identical), and an Arabic numeral designates the individual gene or message and protein [27]. In the set of trees, a multiple sequence alignment was performed using the Muscle method in MEGA7.0 [28]. An ML tree of candidate detoxification genes was constructed by Evoliview [29]. Accession numbers of all protein sequences from other Coleoptera species used in the phylogenetic analysis are listed in Supplementary Materials.

\subsection{Quantitative Real-Time PCR}

We used qRT-PCR to verify the expression of randomly selected candidate T. yunnanensis detoxification genes in different tissues. The cDNA was synthesized with an input of $1 \mu \mathrm{g}$ of total RNA using the Prime ScriptRT Reagent Kit with gDNA Eraser to remove gDNA (AG, Changsha, China). For quantitative real-time PCR (qPCR), various tissues including antennae, heads (without antennae), legs, and carcasses from female and male adults were collected and immediately immersed in liquid nitrogen. qPCR was performed using SYBR Premix EX Taq ${ }^{\mathrm{TM}}$ (AG, Changsha, China) with three technical replicates of each template from three independent biological pools. For the qPCR analysis, the primers (Table S1) were designed by Primer Premier 5.0 [30]. Each reaction contained a total volume of $20 \mu \mathrm{L}$, consisting of $10 \mu \mathrm{L}$ of SYBR Green PCR Master Mix, $0.8 \mu \mathrm{L}$ of each primer $(10 \mu \mathrm{M})$, $2 \mu \mathrm{L}(20 \mathrm{ng})$ of cDNA template, and $6.4 \mu \mathrm{L}$ of nuclease-free water. The $\beta$-actin gene was used as an endogenous control. qPCR cycling parameters were: $94{ }^{\circ} \mathrm{C}$ for $4 \mathrm{~min}$ followed by 40 cycles at $94{ }^{\circ} \mathrm{C}$ for $20 \mathrm{~s}$ and $60^{\circ} \mathrm{C}$ for $30 \mathrm{~s}$. Relative gene expression level was calculated using the Q-GENE statistical analysis package [31].

\section{Results}

\subsection{Transcriptome Assembly}

An Illumina HiSeq platform was used to sequence antennae, head, legs, and carcasses of adult female and male T. yunnanensis transcriptomes. We obtained 61.51 million (MA-1), 47.40 million (MA-2), and 52.92 million (MA-3) raw reads from the antennae of a male adult, 58.15 million (MH-1), 50.67 million (MH-2), and 61.09 million (MH-3) raw-reads from the head of a male adult, and there are raw readings from other organizations in Table 1. Filtering resulted in 58.28 million (MA-1), 46.26 million (MA-2), 51.60 million (MA-3), 55.80 million (MH-1), 49.30 million (MH-2), 59.09 million (MH-3), 55.93 million (ML-1), 53.53 million (ML-2), and 56.48 million (ML-3) clean reads (Table 1). The percentages of reads with Q20 and Q30 values for each library were approximately $98 \%$ and $93 \%$, respectively. The GC content ranged from $38.55 \%$ to $52.60 \%$ (Table 1). The final transcript dataset contained 100,455 unigenes with a mean length of $877 \mathrm{bp}$ and an N50 length of $1153 \mathrm{bp}$ (Table 2), indicating the high quality of our assembly.

\subsection{Functional Annotation of the Unigenes in T. yunnanensis}

A total of 100,455 unigenes were annotated by searching against six databases using BLAST. Specifically, 60,067 (59.79\%) unigenes were matched in the non-redundant (NR) database, which accounted for the largest match. Further, 54,782 (54.53\%) were annotated in the nucleotide (NT) database, 50,102 (49.87\%) were annotated by blasting against the Swiss Port database. Additionally, the euKaryotic Ortholog Groups (KOG) database had the lowest number of annotated unigenes, with 19,808 (19.71\%) unigenes (Table 3). Species with the highest proportion of similar genes were Nephila clavipes $(21.00 \%)$, followed by Dendroctonus ponderosae $(19.2 \%)$, Hyalella azteca $(8.0 \%)$, Toxocara canis $(4.2 \%)$ and Lucilia cuprina (2.1\%) (Figure 1). 
Table 1. Summary of the transcriptome sequencing data from T. yunnanensis.

\begin{tabular}{cccccc}
\hline Sample & Raw Reads & Clean Reads & Q20 (\%) & Q30 (\%) & GC (\%) \\
\hline MA_1 & $615,114,60$ & $582,887,10$ & 97.95 & 94.15 & 47.57 \\
MA_2 & $474,005,30$ & $462,665,14$ & 97.90 & 93.91 & 42.76 \\
MA_3 & $529,246,96$ & $516,082,96$ & 98.06 & 94.27 & 42.81 \\
MH_1 & $581,529,36$ & $558,021,48$ & 97.75 & 93.46 & 38.56 \\
MH_2 & $506,735,06$ & $493,021,74$ & 97.96 & 94.00 & 38.74 \\
MH_3 & $610,987,72$ & $590,914,14$ & 98.04 & 94.16 & 40.09 \\
ML_1 & $582,201,60$ & $559,355,36$ & 98.04 & 94.29 & 45.54 \\
ML_2 & $561,873,52$ & $535,354,48$ & 98.05 & 94.30 & 43.28 \\
ML_3 & $587,793,32$ & $564,878,02$ & 97.88 & 93.93 & 42.65 \\
MC_1 & $640,881,58$ & $628,616,66$ & 97.63 & 93.21 & 38.55 \\
MC_2 & $651,352,06$ & $633,407,70$ & 97.87 & 93.79 & 39.15 \\
MC_3 & $591,512,94$ & $566,883,04$ & 97.10 & 92.16 & 41.95 \\
FA_1 & $523,288,02$ & $510,649,40$ & 98.11 & 94.44 & 46.17 \\
FA_2 & $568,975,58$ & $533,953,70$ & 98.00 & 94.23 & 46.38 \\
FA_3 & $535,300,88$ & $500,120,04$ & 98.00 & 94.24 & 46.14 \\
FH_1 & $513,240,72$ & $502,592,66$ & 97.95 & 93.94 & 39.67 \\
FH_2 & $558,425,54$ & $547,795,96$ & 97.81 & 93.65 & 39.48 \\
FH_3 & $640,915,78$ & $629,613,86$ & 97.62 & 93.18 & 39.24 \\
FL_1 & $586,049,54$ & $572,336,12$ & 97.83 & 93.72 & 43.91 \\
FL_2 & $503,729,98$ & $491,791,08$ & 97.82 & 93.72 & 42.40 \\
FL_3 & $435,955,04$ & $420,544,90$ & 97.94 & 94.19 & 52.60 \\
FC_1 & $572,708,34$ & $560,824,28$ & 97.67 & 93.30 & 40.48 \\
FC_2 & $533,416,24$ & $517,611,84$ & 97.87 & 93.78 & 41.24 \\
FC_3 & $505,727,10$ & $495,473,28$ & 97.77 & 93.55 & 39.88
\end{tabular}

MA: male antennae, MH: male head, ML: male leg, MC: male carcasses, FA: female antennae, FH: female head, FL: female leg, FC: female carcasses.

Table 2. Summary of transcriptome splicing length distribution data from T. yunnanensis.

\begin{tabular}{cccccc}
\hline & Min Length & Mean Length & Max Length & N50 & Total Nucleotides \\
\hline Transcripts & 154 & 1005 & 59,000 & 1465 & $184,171,785$ \\
Genes & 301 & 877 & 59,000 & 1153 & $880,711,34$ \\
\hline
\end{tabular}

Table 3. Statistics of gene annotation success rate.

\begin{tabular}{ccc}
\hline & Number of Genes & Percentage (\%) \\
\hline Annotated in NR & 60,067 & 59.79 \\
Annotated in NT & 54,782 & 54.53 \\
Annotated in SwissProt & 50,102 & 49.87 \\
Annotated in PFAM & 53,205 & 52.96 \\
Annotated in GO & 53,205 & 52.96 \\
Annotated in KOG & 19,808 & 19.71 \\
Annotated in all databases & 1666 & 1.65 \\
Annotated in at least one database & 79,508 & 79.14 \\
Total unigenes & 100,455 & 100 \\
\hline
\end{tabular}

Using a gene ontology (go) method, annotated genes were divided into three categories (a total of 56 functional groups): biological process, cell composition, and molecular function. Among the biological processes, the subcategories cellular process, metabolic process, and single-organism process contained the most unigenes. In the cellular component class, the subcategories cell and cell part contained the most unigenes. Binding and catalytic activity were the most numerous subcategories in the "molecular function" category (Figure 2a). For the euKaryotic Ortholog Groups (KOG) functional classification, we annotated about 19,808 unigenes and divided them into 25 molecular families (Figure 2b). Among them, the largest category was the general function prediction only, followed by amino acid transport and metabolism and energy production and conversion. Cell motility 
and nuclear structure were the smallest groups (Figure 2b). A KEGG analysis was used to classify the annotated genes into different KEGG pathway functional categories (Figure 2c). The most representative pathways were amino acid metabolism, carbohydrate metabolism, overview, and signal transduction (Figure 2c).

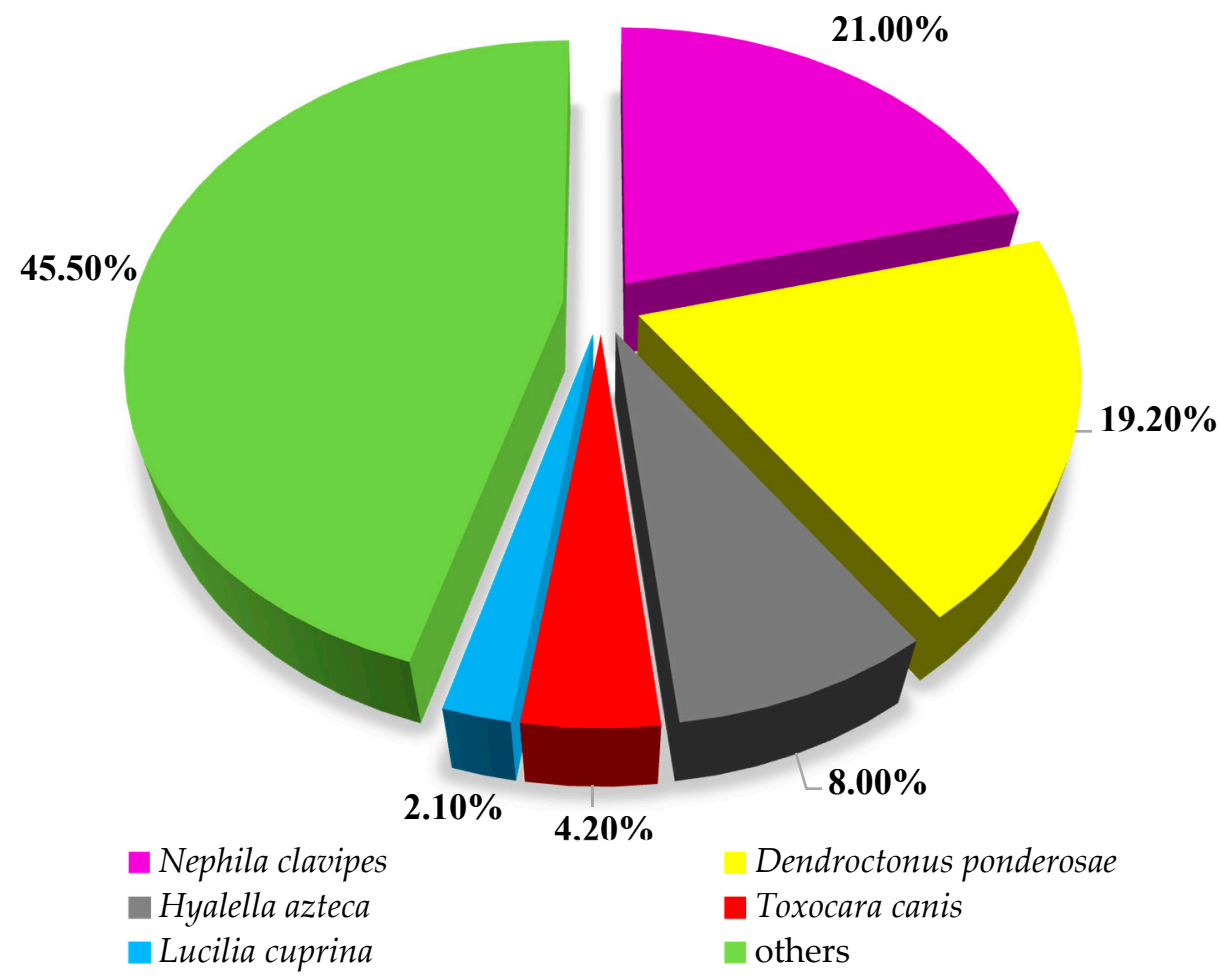

Figure 1. The unigene BLASTx searches against the $\mathrm{Nr}$ database for species distribution analysis.

\subsection{Identification of Candidate CYPS, GSTs, and CCES}

In this study, a total of 51 predicted CYPs transcripts were identified from the transcriptomes of different issues of T. yunnanensis using the BLASTx program. The sequence identities of these candidate CYPs with other Coleoptera insects ranged from $32.69 \%$ to 96.54\% in the NCBI database (Table 4). According to the CYP nomenclature, we classified 51 CYP sequences into four families (CYP2, CYP3, CYP4, mitochondrial CYP). After removing 25 short sequences (aa < 390) [27], 26 sequences with complete open reading frames were used to construct a phylogenetic tree (Figure 3). The phylogenetic tree showed that TyunCYP had a high homology with DponCYP. The largest family was the CYP3 family, which included 13 members and the CYP3 family contained two subfamilies, CYP6 and CYP9. The results of the phylogenetic tree show that five CYPs (TyunCYP6BW2, TyunCYP6DE1, TyunCYP6DF1, TyunCYP6BX1, TyunCYP6DJ1) belong to the CYP6 subfamily. The second largest family is the CYP4 family; we identified eight genes (TyunCYP393A1, TyunCYP6BK1, TyunCYP411A1, TyunCYP349B2, TyunCYP4CV1, TyunCYP4BQ1, TyunCYP4G2, TyunCYP4BG1) belonging to the CYP4 family. 


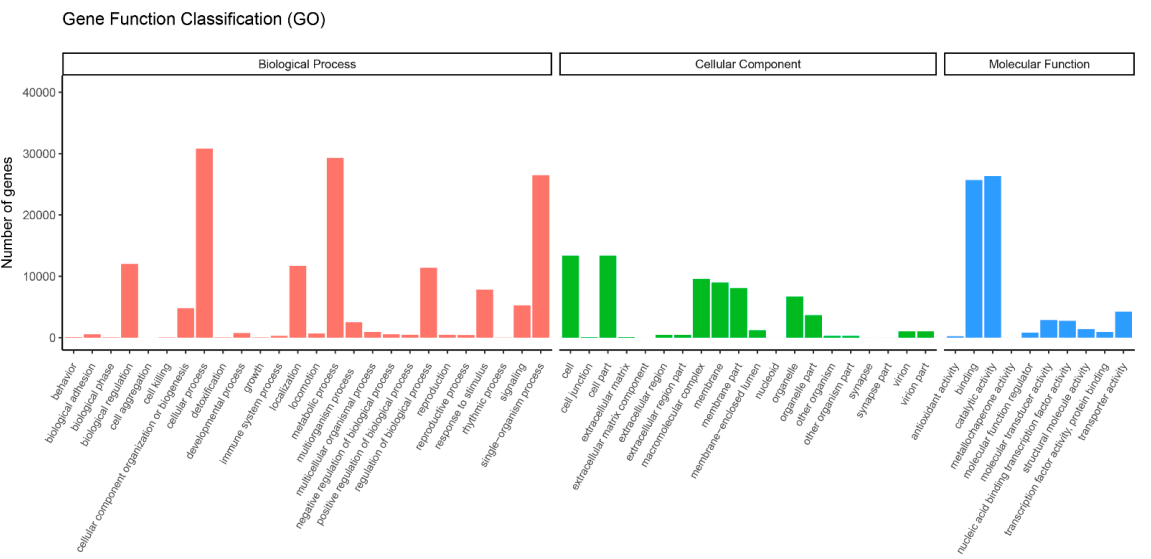

(a)

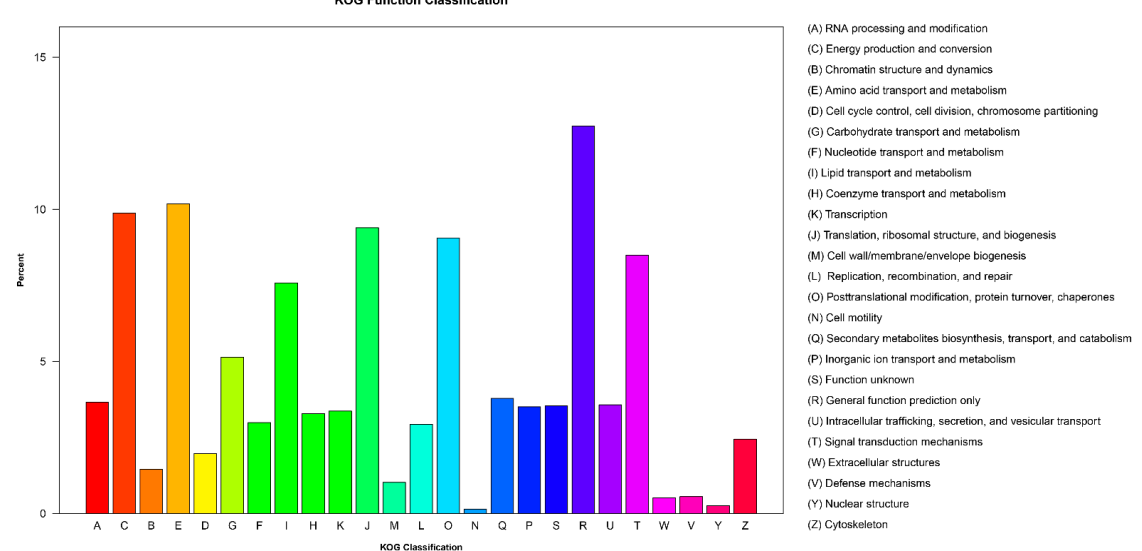

(b)

\section{KEGG Classification}

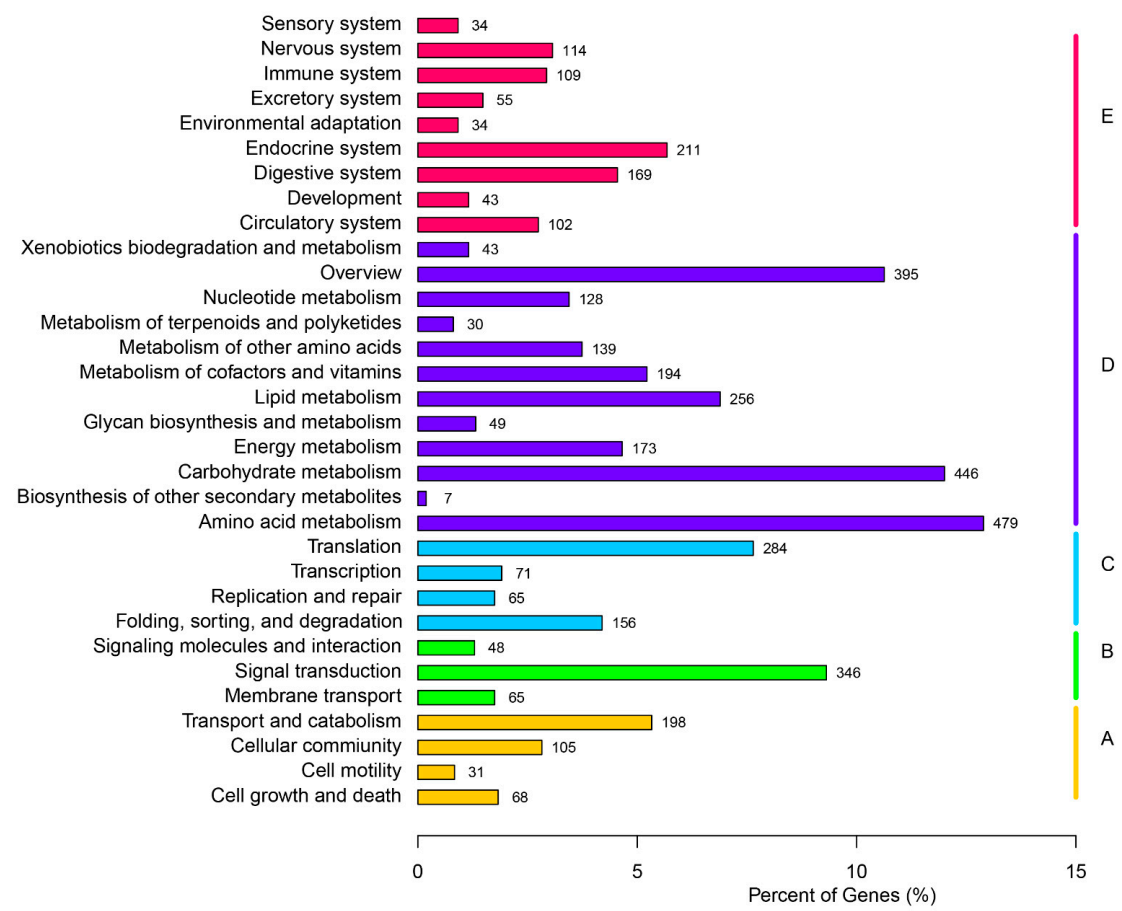

(c)

Figure 2. Results of BLASTx matches of T. yunnanensis transcriptome unigenes, gene ontology, KOG classification, and KEGG pathway annotation. (a) Gene ontology classifications of T. yunnanensis unigenes. (b) KOG classifications of T. yunnanensis unigenes. (c) KEGG classification of T. yunnanensis unigenes. 
Table 4. Best BLASTX matches of T. yunnanensis CYPs.

\begin{tabular}{|c|c|c|c|c|c|c|}
\hline Gene Name & Gene ID & aa & E-Value & Identity & Accession & Species Name \\
\hline TyunCYP349A1 & i2_LQ_TYUN_c39805/f1p0/2021 & 60 & $1 \times 10^{-20}$ & $65.00 \%$ & AEL88544.1 & Dendroctonus rhizophagus \\
\hline TyunCYP9Z1 & i2_LQ_TYUN_c22584/f1p0/2717 & 145 & $5 \times 10^{-82}$ & $82.64 \%$ & AEL88550.1 & Dendroctonus rhizophagus \\
\hline TyunCYP314A1 & i1_LQ_TYUN_c152368/f1p0/1050 & 147 & $6 \times 10^{-78}$ & $83.33 \%$ & AFI45004.1 & Dendroctonus ponderosae \\
\hline TyunCYP347B1 & i4_LQ_TYUN_c2131/f1p0/4666 & 447 & 0 & $81.94 \%$ & AFI45010.1 & Dendroctonus ponderosae \\
\hline TyunCYP410C1 & Cluster-27834.11928 & 456 & $1 \times 10^{-107}$ & $39.43 \%$ & AFI45013.1 & Dendroctonus ponderosae \\
\hline TyunCYP4CV1 & Cluster-27834.3883 & 507 & 0 & $77.47 \%$ & AFI45020.1 & Dendroctonus ponderosae \\
\hline TyunCYP6BW1 & i3_LQ_TYUN_c10935/f1p0/4006 & 283 & $9 \times 10^{-180}$ & $85.16 \%$ & AFI45024.1 & Dendroctonus ponderosae \\
\hline TyunCYP6BW2 & i1_HQ_TYUN_c416/f4p1/1768 & 506 & 0 & $86.76 \%$ & AFI45026.1 & Dendroctonus ponderosae \\
\hline TyunCYP6BX1 & Cluster-27834.4019 & 478 & 0 & $71.43 \%$ & AFI45028.1 & Dendroctonus ponderosae \\
\hline TyunCYP6DJ1 & i1_LQ_TYUN_c33388/f1p0/1763 & 507 & 0 & $70.71 \%$ & AFI45041.1 & Dendroctonus ponderosae \\
\hline TyunCYP9Z4 & i1_LQ_TYUN_c78340/f1p0/1911 & 526 & 0 & $84.22 \%$ & AFI45045.1 & Dendroctonus ponderosae \\
\hline TyunCYP6DJ2 & i2_LQ_TYUN_c23405/f1p0/2443 & 66 & $6 \times 10^{-7}$ & $67.65 \%$ & AGF69211.1 & Dendroctonus valens \\
\hline TyunCYP4G1 & i3_LQ_TYUN_c4999/f1p0/3248 & 242 & $5 \times 10^{-158}$ & $93.80 \%$ & ALD15896.1 & Dendroctonus armandi \\
\hline TyunCYP305F1 & Cluster-27834.3827 & 491 & 0 & $87.53 \%$ & ALD15904.1 & Dendroctonus armandi \\
\hline TyunCYP352B1 & i2_LQ_TYUN_c27719/f1p0/2254 & 225 & $4 \times 10^{-74}$ & $69.36 \%$ & ALD15909.1 & Dendroctonus armandi \\
\hline TyunCYP4BD4V & i1_LQ_TYUN_c15852/f1p0/1569 & 166 & $8 \times 10^{-68}$ & $67.31 \%$ & ALD15912.1 & Dendroctonus ponderosae \\
\hline TyunCYP6DF1 & Cluster-27834.8537 & 505 & 0 & $72.31 \%$ & ALD15922.1 & Dendroctonus armandi \\
\hline TyunCYP9Z2 & i2_LQ_TYUN_c15205/f1p0/2172 & 575 & 0 & $81.30 \%$ & ALD15924.1 & Dendroctonus armandi \\
\hline TyunCYP434A1 & Cluster-27834.7270 & 371 & $7 \times 10^{-147}$ & $71.53 \%$ & XP_019754109.1 & Dendroctonus ponderosae \\
\hline TyunCYP4BQ1 & Cluster-27834.7070 & 397 & 0 & $67.84 \%$ & XP_019754731.1 & Dendroctonus ponderosae \\
\hline TyunCYP6DE1 & i1_HQ_TYUN_c29325/f3p0/1840 & 509 & 0 & $76.23 \%$ & XP_019755300.1 & Dendroctonus ponderosae \\
\hline TyunCYP315A1 & Cluster-27834.2194 & 292 & $8 \times 10^{-139}$ & $72.26 \%$ & XP_019755328.1 & Dendroctonus ponderosae \\
\hline TyunCYP315A2 & Cluster-27834.2193 & 208 & $2 \times 10^{-20}$ & $70.15 \%$ & XP_019755336.1 & Dendroctonus ponderosae \\
\hline TyunCYP4G2 & i1_HQ_TYUN_c24270/f27p4/1844 & 563 & 0 & $92.11 \%$ & XP_019755432.1 & Dendroctonus ponderosae \\
\hline TyunCYP4BG1 & Cluster-27834.11078 & 501 & 0 & $78.09 \%$ & XP_019756327.1 & Dendroctonus ponderosae \\
\hline TyunCYP410A1 & i1_LQ_TYUN_c6617/f1p1/1851 & 273 & $5 \times 10^{-82}$ & $53.52 \%$ & XP_019756499.1 & Dendroctonus ponderosae \\
\hline TyunCYP411A1 & i2_LQ_TYUN_c22094/f1p0/2786 & 490 & 0 & $76.22 \%$ & XP_019758316.1 & Dendroctonus ponderosae \\
\hline TyunCYP411A2 & i2_LQ_TYUN_c11742/f1p1/2726 & 185 & $6 \times 10^{-77}$ & $71.43 \%$ & XP_019758318.1 & Dendroctonus ponderosae \\
\hline TyunCYP345F1 & Cluster-26110.0 & 500 & 0 & $82.20 \%$ & XP_019759785.1 & Dendroctonus ponderosae \\
\hline TyunCYP307B1 & Cluster-27834.15214 & 399 & $1 \times 10^{-153}$ & $77.06 \%$ & XP_019760073.1 & Dendroctonus ponderosae \\
\hline TyunCYP307A1 & i3_LQ_TYUN_c19449/f1p0/3461 & 492 & 0 & $96.54 \%$ & XP_019760634.1 & Dendroctonus ponderosae \\
\hline TyunCYP393A2 & i0_LQ_TYUN_c4806/f1p0/966 & 112 & $5 \times 10^{-17}$ & $71.93 \%$ & XP_019760717.1 & Dendroctonus ponderosae \\
\hline TyunCYP9AP1 & i1_LQ_TYUN_c81629/f1p2/1918 & 483 & 0 & $72.57 \%$ & XP_019760793.1 & Dendroctonus ponderosae \\
\hline TyunCYP9AN1 & i2_LQ_TYUN_c15846/f1p0/2129 & 523 & 0 & $79.31 \%$ & XP_019761014.1 & Dendroctonus ponderosae \\
\hline TyunCYP433A1 & i1_LQ_TYUN_c80202/f1p0/1820 & 274 & $2 \times 10^{-128}$ & $70.45 \%$ & XP_019761671.1 & Dendroctonus ponderosae \\
\hline TyunCYP9Z3 & i2_LQ_TYUN_c27749/f1p0/2479 & 151 & $2 \times 10^{-44}$ & $73.33 \%$ & XP_019764934.1 & Dendroctonus ponderosae \\
\hline TyunCYP345E1 & i1_HQ_TYUN_c62660/f2p0/1795 & 304 & $4 \times 10^{-159}$ & $72.33 \%$ & XP_019765603.1 & Dendroctonus ponderosae \\
\hline TyunCYP347D1 & Cluster-20450.0 & 498 & 0 & $78.93 \%$ & XP_019767652.1 & Dendroctonus ponderosae \\
\hline TyunCYP303A1 & Cluster-27834.21448 & 484 & 0 & $76.80 \%$ & XP_019768066.1 & Dendroctonus ponderosae \\
\hline TyunCYP18A1 & i2_LQ_TYUN_c27030/f1p0/2265 & 159 & $8 \times 10^{-82}$ & $79.87 \%$ & XP_019768143.1 & Dendroctonus ponderosae \\
\hline TyunCYP18A2 & i2_HQ_TYUN_c25448/f2p0/2291 & 525 & 0 & $90.50 \%$ & XP_019768147.1 & Dendroctonus ponderosae \\
\hline TyunCYP393A1 & Cluster-27834.3112 & 461 & 0 & $69.78 \%$ & XP_019768810.1 & Dendroctonus ponderosae \\
\hline TyunCYP334E1 & Cluster-27834.2023 & 583 & 0 & $85.74 \%$ & XP_019770320.1 & Dendroctonus ponderosae \\
\hline TyunCYP347E1 & i2_LQ_TYUN_c3851/f1p0/2279 & 500 & 0 & $79.00 \%$ & XP_019771168.1 & Dendroctonus armandi \\
\hline TyunCYP6DG3 & i3_LQ_TYUN_c17722/f1p0/3197 & 354 & 0 & $77.68 \%$ & XP_019771861.1 & Dendroctonus ponderosae \\
\hline TyunCYP9AZ1 & i2_LQ_TYUN_c28708/f1p0/2957 & 134 & $2 \times 10^{-70}$ & $79.85 \%$ & XP_019773084.1 & Dendroctonus ponderosae \\
\hline TyunCYP334E2 & i2_LQ_TYUN_c27105/f1p0/2563 & 498 & 0 & $83.77 \%$ & XP_019773300.1 & Dendroctonus ponderosae \\
\hline TyunCYP349B2 & Cluster-40684.0 & 420 & 0 & $82.86 \%$ & XP_019773560.1 & Dendroctonus ponderosae \\
\hline TyunCYP434A2 & i1_HQ_TYUN_c939/f2p0/1483 & 360 & $5 \times 10^{-124}$ & $50.00 \%$ & XP_019869691.1 & Aethina tumida \\
\hline TyunCYP6BW3 & i2_LQ_TYUN_c30164/f1p0/2137 & 382 & $2 \times 10^{-121}$ & $48.28 \%$ & XP_023014703.1 & Leptinotarsa decemlineata \\
\hline TyunCYP410C1 & i0_LQ_TYUN_c9810/f1p0/524 & 150 & $8 \times 10^{-12}$ & $32.69 \%$ & XP_030755100.1 & Sitophilus oryzae \\
\hline
\end{tabular}

A total of 33 candidate GSTs were identified from the total transcriptome of the different developmental stages of T. yunnanensis. The sequence identities of these candidate GSTs with other dipteran insects ranged from $51.96 \%$ to $97.14 \%$ in the NCBI database (Table 5). After removing short sequences (aa < 150) [32], 18 GSTs were chosen for the phylogenetic analysis. A neighbor-joining tree was subsequently constructed using our identified putative GST proteins and the sequences from four other Coleoptera species, D. ponderosae, T. castaneum, D. valens, and A. planipennis (Figure 4). Ten GSTs were identified in the transcriptome of T. yunnanensis, including six delta/epsilon class GSTs, four omega class GST, five sigma class GSTs, two theta class GSTs, and one microsomal class GST. The phylogenetic tree showed that TyunGST had a high homology with DarmGST. 


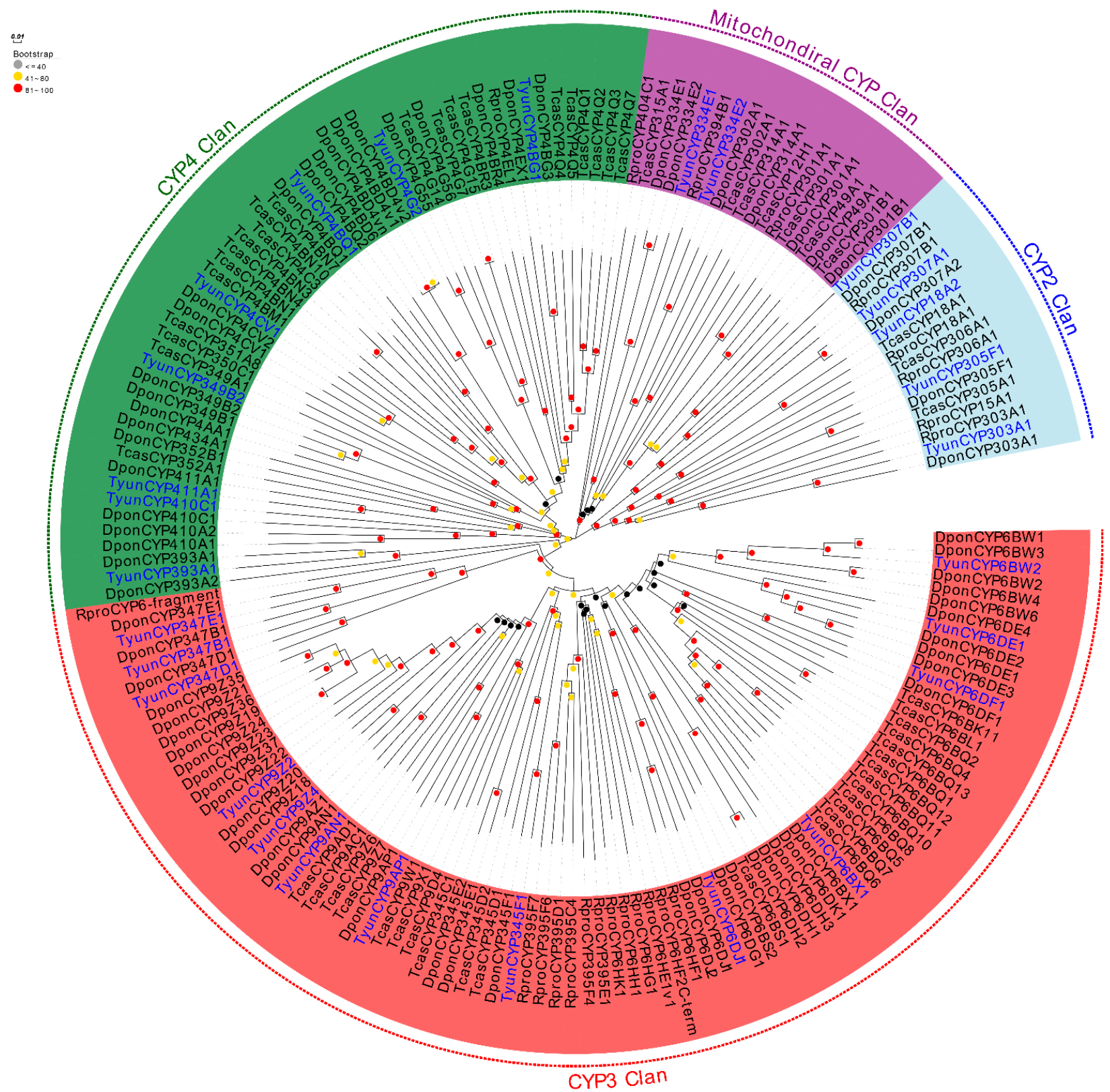

Figure 3. Neighbor-joining tree of candidate CYPs. Bootstrap values after 1000 replications. Dpon, Dendroctonus ponderosae; Rpro, Rhodnius prolixus; Tcas, Tribolium castaneum; Tyun, Tomicus yunnanensis.

We identified 29 transcripts encoding CCEs in the T. yunnanensis transcriptome by a bioinformatics analysis and all of them were more conserved across other CCEs variants, with 42.86 to $93.03 \%$ amino acid identity (Table 6). To guarantee the reliability of the phylogenetic tree, 13 CCEs encoding short sequences wereremoved (aa < 480) [33], and 16 CCEs in our transcriptomes were aligned with CCEs from other coleoptera species (Figure 5). A total of 16 CCE genes were identified in the transcriptome of T. yunnanensis, including nine xenobiotic metabolizing enzymes class CCE, three microsomal and alphaesterases class CCE, two beta- and pheromone esterases class CCE, one JHE class CCE, and one $\mathrm{CO}$ class CCE. 
Table 5. Best BLASTX matches of T. yunnanensis GSTs.

\begin{tabular}{|c|c|c|c|c|c|c|}
\hline Gene Name & Gene ID & aa & E-Value & Identity & Accession & Species Name \\
\hline TyunGST1 & i2_LQ_TYUN_c36781/f1p0/2077 & 217 & $2 \times 10^{-145}$ & $91.71 \%$ & AIC76455.1 & Dendroctonus armandi \\
\hline TyunGST2 & Cluster-27834.9472 & 70 & $6 \times 10^{-41}$ & $97.14 \%$ & AJE61306.1 & Dendroctonus armandi \\
\hline TyunGST3 & i0_LQ_TYUN_c12133/f1p0/748 & 141 & $3 \times 10^{-48}$ & $57.25 \%$ & AJE61307.1 & Dendroctonus armandi \\
\hline TyunGST4 & i2_LQ_TYUN_c18657/f1p0/2385 & 112 & $3 \times 10^{62}$ & $76.74 \%$ & AJE61308.1 & Dendroctonus armandi \\
\hline TyunGST5 & i3_LQ_TYUN_c11135/f1p0/3582 & 79 & $5 \times 10^{-33}$ & $82.35 \%$ & AJE61309.1 & Dendroctonus armandi \\
\hline TyunGST6 & Cluster-27834.6624 & 230 & $2 \times 10^{-146}$ & $83.48 \%$ & AJE61311.1 & Dendroctonus armandi \\
\hline TyunGST7 & Cluster-27834.8595 & 244 & $8 \times 10^{-160}$ & $88.52 \%$ & AJE61312.1 & Dendroctonus armandi \\
\hline TyunGST8 & i1_LQ_TYUN_c149780/f1p1/1152 & 245 & $2 \times 10^{-150}$ & $82.04 \%$ & AVR54955.1 & Sitophilus oryzae \\
\hline TyunGST9 & i3_LQ_TYUN_c12086/f1p0/3529 & 218 & $2 \times 10^{-142}$ & $88.58 \%$ & AVR54957.1 & Sitophilus oryzae \\
\hline TyunGST10 & i2_LQ_TYUN_c14505/f1p9/2537 & 52 & $6 \times 10^{-15}$ & $71.43 \%$ & AVR54966.1 & Sitophilus oryzae \\
\hline TyunGST11 & Cluster-27834.9551 & 105 & $9 \times 10^{-41}$ & $64.42 \%$ & AVT42177.1 & Lissorhoptrus oryzophilus \\
\hline TyunGST12 & i0_LQ_TYUN_c7185/f1p0/508 & 148 & $6 \times 10^{-48}$ & $62.50 \%$ & AVT42178.1 & Lissorhoptrus oryzophilus \\
\hline TyunGST13 & i2_LQ_TYUN_c7650/f1p0/2527 & 118 & $3 \times 10^{-64}$ & $87.85 \%$ & AVT42182.1 & Lissorhoptrus oryzophilus \\
\hline TyunGST14 & Cluster-27834.21190 & 219 & $3 \times 10^{-69}$ & $51.96 \%$ & AVT42185.1 & Lissorhoptrus oryzophilus \\
\hline TyunGST15 & i2_LQ_TYUN_c8057/f1p0/2856 & 294 & $7 \times 10^{-150}$ & $67.12 \%$ & AVT42197.1 & Lissorhoptrus oryzophilus \\
\hline TyunGST16 & Cluster-27834.9541 & 219 & $1 \times 10^{-144}$ & $89.50 \%$ & QFU14637.1 & Dendroctonus armandi \\
\hline TyunGST17 & i4_LQ_TYUN_c8880/f1p0/4497 & 137 & $3 \times 10^{-69}$ & $93.69 \%$ & QFFU14640.1 & Dendroctonus armandi \\
\hline TyunGST18 & Cluster-1630.0 & 205 & $1 \times 10^{-76}$ & $56.78 \%$ & QFFU14643.1 & Dendroctonus armandi \\
\hline TyunGST19 & i2_LQ_TYUN_c12200/f1p0/2343 & 231 & $3 \times 10^{-137}$ & $77.92 \%$ & Q̄FU14646.1 & Dendroctonus armandi \\
\hline TyunGST20 & i3_LQ_TYUN_c2494/f1p0/3326 & 218 & $4 \times 10^{-146}$ & $90.41 \%$ & XP_019753833.1 & Dendroctonus ponderosae \\
\hline TyunGST21 & i2_LQ_TYUN_c34577/f1p0/2723 & 78 & $7 \times 10^{-41}$ & $83.54 \%$ & XP_019753835.1 & Dendroctonus ponderosae \\
\hline TyunGST22 & i2_LQ_TYUN_c23261/f1p0/2987 & 124 & $2 \times 10^{-73}$ & $93.86 \%$ & XP_019755130.1 & Dendroctonus ponderosae \\
\hline TyunGST23 & i2_LQ_TYUN_c24320/f1p0/2660 & 150 & $2 \times 10^{-35}$ & $84.72 \%$ & XP_019755704.1 & Dendroctonus ponderosae \\
\hline TyunGST24 & i7_LQ_TYUN_c267/f1p0/7646 & 72 & $7 \times 10^{-22}$ & $67.21 \%$ & XP_019755792.1 & Dendroctonus ponderosae \\
\hline TyunGST25 & i3_LQ_TYUN_c4401/f1p0/3564 & 111 & $4 \times 10^{-53}$ & $86.27 \%$ & XP_019755793.1 & Dendroctonus ponderosae \\
\hline TyunGST26 & i3_LQ_TYUN_c26609/f1p0/3037 & 203 & $3 \times 10^{107}$ & $72.00 \%$ & XP_019755830.1 & Dendroctonus ponderosae \\
\hline TyunGST27 & i0_LQ_TYUN_c24616/f1p0/906 & 205 & $1 \times 10^{-129}$ & $85.37 \%$ & XP_019760728.1 & Dendroctonus ponderosae \\
\hline TyunGST28 & i2_LQ_TYUN_c11257/f1p0/2424 & 559 & 0 & $75.62 \%$ & XP_019764595.1 & Dendroctonus ponderosae \\
\hline TyunGST29 & Cluster-27834.9273 & 224 & $4 \times 10^{-124}$ & $73.66 \%$ & XP_019766435.1 & Dendroctonus ponderosae \\
\hline TyunGST30 & i1_LQ_TYUN_c146891/f1p0/1112 & 237 & $3 \times 10^{-158}$ & $89.83 \%$ & XP_019768505.1 & Dendroctonus ponderosae \\
\hline TyunGST31 & i0_LQ_TYUN_c13481/f1p0/385 & 70 & $4 \times 10^{-32}$ & $82.86 \%$ & XP_019770972.1 & Dendroctonus ponderosae \\
\hline TyunGST32 & i1_LQ_TYUN_c153362/f1p1/1072 & 206 & $3 \times 10^{-77}$ & $57.28 \%$ & XP_023313068.1 & Anoplophora glabripennis \\
\hline TyunGST33 & i1_LQ_TYUN_c150961/f1p1/1003 & 206 & $2 \times 10^{-78}$ & $58.54 \%$ & XP_023313069.1 & Anoplophora glabripennis \\
\hline
\end{tabular}

\subsection{Tissue Expression Profile of the CYP, GST, and CCE Genes}

In order to explore the expression profiles of detoxification genes in different tissues, we screened all 113 genes from the DEG library. In total, 64 genes were found, and 49 genes were missing from the DEG data. The expression profiles based on FPKM values revealed that several detoxification genes (TyunCYP410A1, TyunCYP9AN1, TyunCYP393A1, TyunGST22, TyunGST29, TyunGST31, TyunCCE8, and TyunCCE17) are highly expressed in the antenna (FPKM > 100) and the expression levels of these genes are all higher in male antennae than in female antennae (Figure 6). TyunCYP4G2 is highly expressed in the residue. Tyun315A1, TyunCYP6BK1, TyunCYP6DF1, and TyunGST3 are expressed in all tissues; TyunCYP6DF1 expression in antennae is higher than in other tissues. TyunCYP345E1 and TyunCYP345F1 are little expressed in all tissues (Figure 6a). TyunGST11 and TyunGST33 are expressed in all tissues, the expression of TyunGST11 in foot and antennae is higher than other tissues, and the expression level of male feet is higher than that of female legs. The antenna expression of TyunGST33 is significantly higher than that of other tissues and the male antenna expression is higher than that of the female (Figure 6b). TyunCCE21 is expressed in all tissues. TyunCCE16 is strongly expressed in the head and carcasses, and expression in these two parts of the females is higher than that of the males (Figure 6c). 


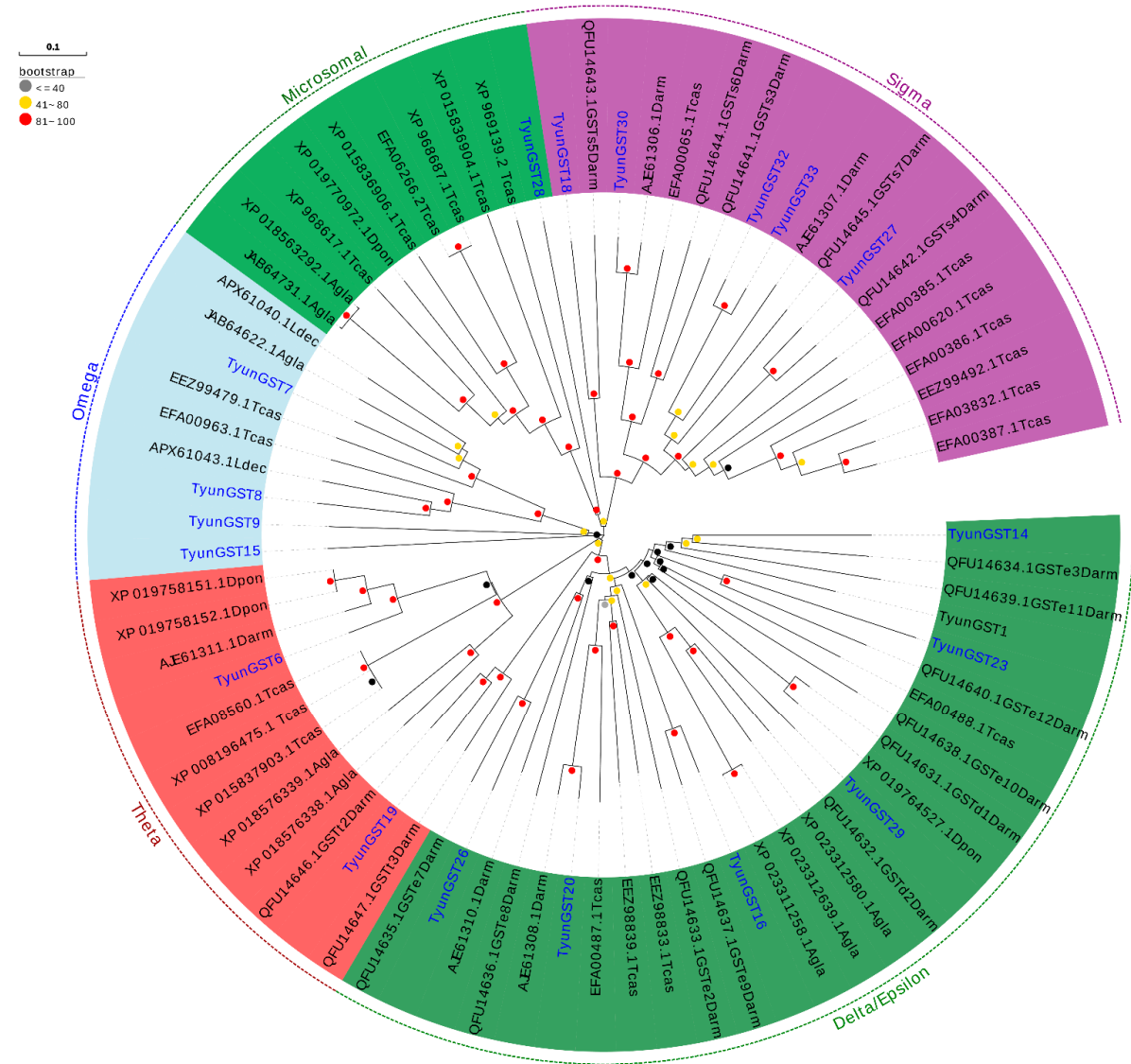

Figure 4. Neighbor-joining tree of candidate GSTs. Bootstrap values after 1000 replications. Dpon, Dendroctonus ponderosae; Darm, Dendroctonus armandi; Agla, Anoplophora glabripennis; Ldec Leptinotarsa decemlineata; Tcas, Tribolium castaneum; Tyun, Tomicus yunnanensis.

Table 6. Best BLASTX matches of T. yunnanensis CCEs.

\begin{tabular}{|c|c|c|c|c|c|c|}
\hline Gene Name & & aa & E-Value & Identity & Accession & Species Name \\
\hline TyunCCE1 & i2_LQ_TYUN_c19726/f1p0/2240 & 580 & 0 & $79.41 \%$ & AYN64423.1 & Dendroctonus armandi \\
\hline TyunCCE2 & i1_LQ_TYUN_c11797/f1p0/1951 & 405 & 0 & $93.03 \%$ & AYN64424.1 & Dendroctonus armandi \\
\hline TyunCCE3 & Cluster-27834.6014 & 133 & $3 \times 10^{-43}$ & $61.42 \%$ & AYN64428.1 & Dendroctonus armandi \\
\hline TyunCCE4 & Cluster-27834.16668 & 243 & $5 \times 10^{-156}$ & $88.48 \%$ & AYN64429.1 & Dendroctonus armandi \\
\hline TyunCCE5 & Cluster-27834.3878 & 550 & $5 \times 10^{-164}$ & $45.34 \%$ & XP_019754206.1 & Dendroctonus ponderosae \\
\hline TyunCCE6 & Cluster-27834.11182 & 570 & 0 & $81.05 \%$ & XP_019754592.1 & Dendroctonus ponderosae \\
\hline TyunCCE7 & Cluster-27834.18917 & 577 & 0 & $87.19 \%$ & XP_019755320.1 & Dendroctonus ponderosae \\
\hline TyunCCE8 & i1_LQ_TYUN_c11402/f1p0/1940 & 563 & 0 & $58.70 \%$ & XP_019755320.1 & Dendroctonus ponderosae \\
\hline TyunCCE9 & Cluster-27834.6015 & 557 & 0 & $64.16 \%$ & XP_019755963.1 & Dendroctonus ponderosae \\
\hline TyunCCE10 & i1_HQ_TYUN_c4678/f4p0/1715 & 558 & 0 & $82.08 \%$ & XP_019756055.1 & Dendroctonus ponderosae \\
\hline TyunCCE11 & i1_LQ_TYUN_c2026/f1p0/1764 & 559 & 0 & $85.15 \%$ & XP_019756056.1 & Dendroctonus ponderosae \\
\hline TyunCCE12 & Cluster-27834.3444 & 122 & $5 \times 10^{-75}$ & $90.98 \%$ & XP_019756397.1 & Dendroctonus ponderosae \\
\hline TyunCCE13 & Cluster-27834.5714 & 268 & $2 \times 10^{-169}$ & $88.01 \%$ & XP_019756739.1 & Dendroctonus ponderosae \\
\hline TyunCCE14 & i1_LQ_TYUN_c29743/f1p0/1703 & 545 & 0 & $50.54 \%$ & XP_019757154.1 & Dendroctonus ponderosae \\
\hline TyunCCE15 & i1_LQ_TYUN_c35851/f1p0/1947 & 228 & $8 \times 10^{-114}$ & $75.60 \%$ & XP_019758307.1 & Dendroctonus ponderosae \\
\hline TyunCCE16 & i1_LQ_TYUN_c30292/f1p1/1995 & 566 & 0 & $88.18 \%$ & XP_019761960.1 & Dendroctonus ponderosae \\
\hline TyunCCE17 & i1_LQ_TYUN_c16028/f1p0/1766 & 347 & 0 & $81.23 \%$ & XP_019764466.1 & Dendroctonus ponderosae \\
\hline TyunCCE18 & i1_LQ_TYUN_c9615/f1p0/1928 & 532 & 0 & $76.15 \%$ & XP_019765893.1 & Dendroctonus ponderosae \\
\hline TyunCCE19 & i1_LQ_TYUN_c37855/f1p0/1526 & 414 & 0 & $83.09 \%$ & XP_019766062.1 & Dendroctonus ponderosae \\
\hline TyunCCE20 & Cluster-27834.3692 & 531 & 0 & $85.50 \%$ & XP_019766553.1 & Dendroctonus ponderosae \\
\hline TyunCCE21 & Cluster-27834.10155 & 569 & 0 & $86.12 \%$ & XP_019769801.1 & Dendroctonus ponderosae \\
\hline TyunCCE22 & i3_LQ_TYUN_c17625/f1p0/3579 & 266 & $2 \times 10^{-136}$ & $89.52 \%$ & XP_019769801.1 & Dendroctonus ponderosae \\
\hline TyunCCE23 & i1_LQ_TYUN_c34869/f1p0/1995 & 577 & 0 & $69.46 \%$ & XP_019769830.1 & Dendroctonus ponderosae \\
\hline TyunCCE24 & Cluster-27834.16736 & 569 & 0 & $75.66 \%$ & XP_019772474.1 & Dendroctonus ponderosae \\
\hline TyunCCE25 & i2_LQ_TYUN_c30644/f1p0/2041 & 337 & 0 & $78.34 \%$ & XP_019773718.1 & Dendroctonus ponderosae \\
\hline TyunCCE26 & Cluster-27834.18773 & 65 & $9 \times 10^{-7}$ & $42.86 \%$ & XP_023727217.2 & Cryptotermes secundus \\
\hline TyunCCE27 & Cluster-27834.13066 & 308 & $7 \times 10^{-127}$ & $60.06 \%$ & XP_030749177.1 & Sitophilus oryzae \\
\hline TyunCCE28 & Cluster-27834.7029 & 572 & 0 & $47.51 \%$ & XP_030754488.1 & Sitophilus oryzae \\
\hline TyunCCE29 & i2_LQ_TYUN_c24874/f1p0/2585 & 308 & $3 \times 10^{-107}$ & $55.89 \%$ & XP_030768230.1 & Sitophilus oryzae \\
\hline
\end{tabular}




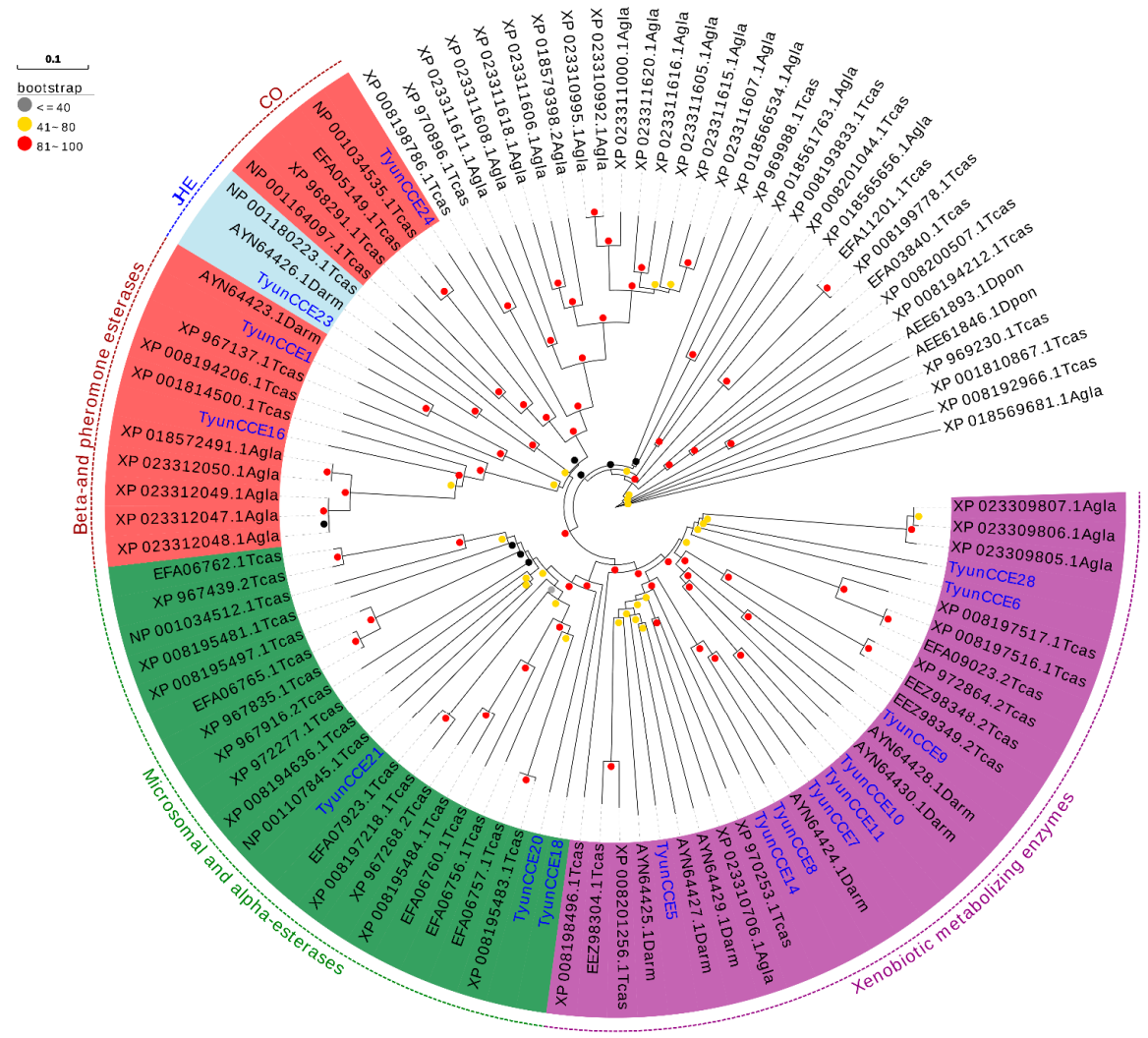

Figure 5. Neighbor-joining tree of candidate CCEs. Bootstrap values after 1000 replications. Dpon, Dendroctonus ponderosae; Darm, Dendroctonus armandi; Agla, Anoplophora glabripennis; Tcas, Tribolium castaneum; Tyun, Tomicus yunnanensis.

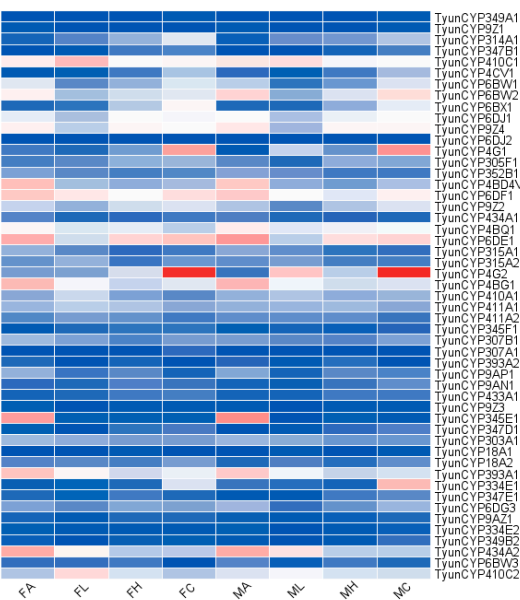

(a)

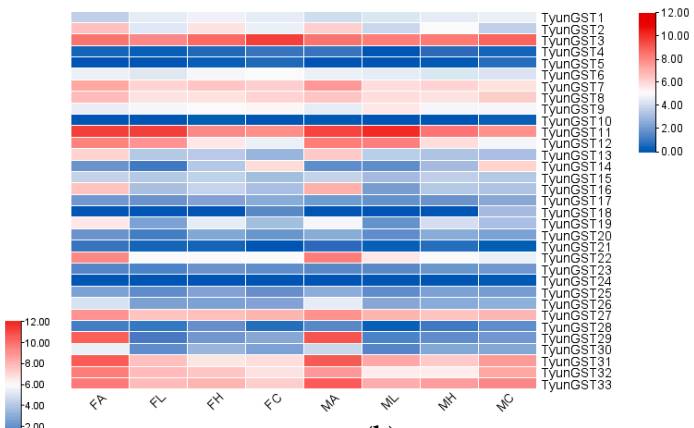

(b)

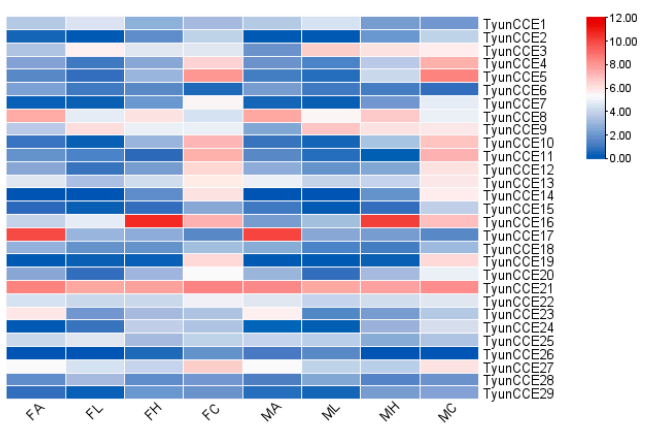

(c)

Figure 6. Expression profiles of detoxification genes in T. yunnanensis. (a) CYP; (b) GST; (c) CCE. MA: male antennae, MH: male head, ML: male legs, MC: male carcasses, FA: female antennae, FH: female head, FL: female legs, FC: female carcasses. 
Further, qPCR was employed to validate the expression of some detoxification genes and to investigate their expression profiles. The qPCR results of TyunCYP4G2, TyunCYP6DF1, TyunGST11, TyunGST33, TyunCCE16, and TyunCCE17 are consistent with the FPKM value analysis results, but the qPCR results of TyunCCE21 differ from the FPKM value analysis, which may be caused by the inconsistency between the qPCR analysis samples and the sequencing samples (Figure 7).

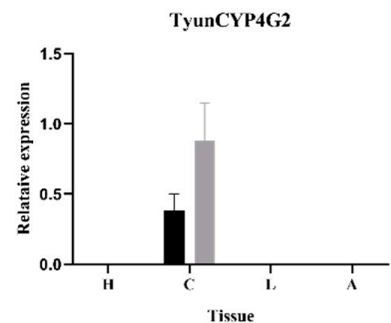

TyunCCE17
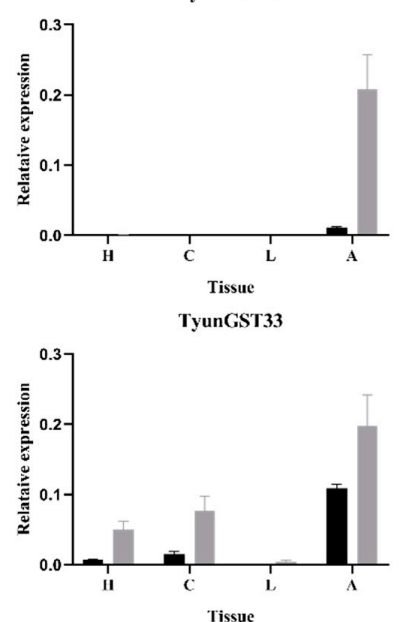

TyunCYP6DF1

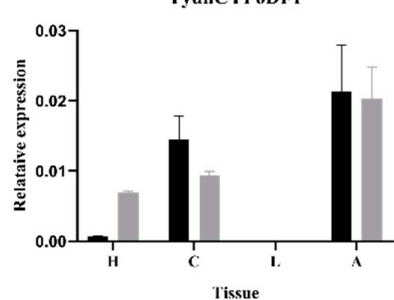

TyunCCE21

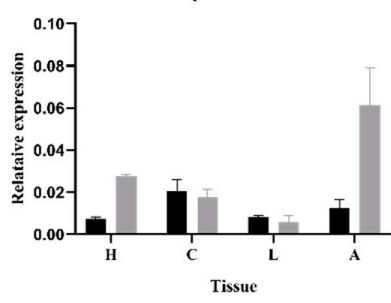

Female

Male

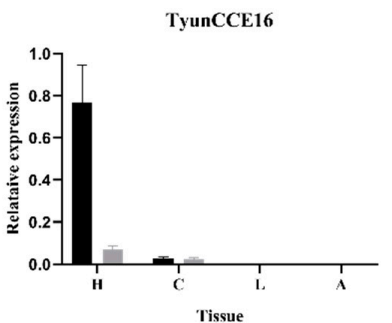

TyunGST11

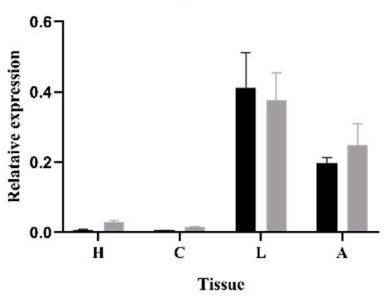

Figure 7. qPCR analysis of T. yunnanensis detoxification genes transcript levels in different tissues. H: head; C: carcasses; L: leg; A: antenna.

\section{Discussion}

Tomicus yunnanensis is one of the most important pests of Pinus yunnanensis. In most insect species, detoxification proteins play a key role in the degradation of plant secondary metabolites. In order to better understand the clues of how insects degrade plant secondary metabolites, we first identified candidate detoxification proteins in the transcripts of the antennae, heads, legs, and carcasses of T. yunnanensis, and studied some of the detoxification proteins and the expression profiles of four different organizations. Our research results provide new evidence for the molecular basis of the detoxification proteins of T. yunnanensis in the metabolic function of detoxification, which may help to develop better methods to control this pest. The identification of at least $51 \mathrm{CYP}$ genes placed T. yunnanensis within the middle of the range of the P450 gene family size in insects for which the genome has been sequenced, ranging from a low of 46 in the honeybee (Apis mellifera Linnaeus, 1758 (Hymenoptera: Apidae)) to 143 in T. castaneum [34], and it was similar to the mountain pine beetle (D. armandi), which has 64 CYPs. Previous studies on T. yunnanensis were mainly in the direction of olfactory-related proteins, but there are few reports on the detoxification genes of T. yunnanensis [35]. In this study, 51 TyunCYPs were analyzed in different adult tissue transcripts, and we found that seven TyunCYPs are predominantly expressed in the antennae of both sexes, belonging to three CYP families, CYP2: TyunCYP305F1; mitochondrial CYP clan: TyunCYP315A1; CYP4: TyunCYP410A1, TyunCYP393A1, and TyunCYP305F1. CYP345E2, a member of CYP3, is an antenna-specific CYP from $D$. pondersae that has been proved to catalyze the oxidation of 
monoterpene volatile compounds in pine hosts [36]. In our study, we found two CYP345E2 homologues, TyunCYP345E1 and TyunCYP345F1 in T. yunnanensis, but the expression levels of TyunCYP345E1 and TyunCYP345F1 in the antennae were both low, which may be caused by differences between species. Some members of the CYP4 family are also involved in odor degradation, and most of them are involved in the synergistic reaction of detoxification and pheromone synthesis [37]. The expression level of TyunCYP393A1 in the antennae of females was higher than that of males, which might be related to the host location of females, while the expression level of TyunCYP410A1 in the antennae of males was higher than that of females, which may be because the male needs to degrade the secondary metabolites produced by the plant defense mechanism. TyunCYP4G2 is specifically and highly expressed in the residue, which may be related to the metabolism of toxic substances in T. yunnanensis. In the CYP6 family, few members have been reported to have odorant clearance functions [38]. In many (but not all) studies, genes from the CYP6 subfamily are shown to metabolize xenobiotics and plant natural compounds [27]. Several genes have been reported to have specific expression in the olfactory organs, such as CYP6B48, CYP6B42, and CYP6AE49 expressed in the male and female antennae of Spodoptera litura [39]. PxCYP6BG3 and PxCYP6BG6 were found in Plutella xylostell, which may be related to larval odor clearance [40]. In this study, we did not find any genes that were specifically and highly expressed in the olfactory organs of the CYP6 family.

The functions of GSTs in many insects' physiological functions, such as insecticide resistance and detoxification (plant secondary metabolites) have been fully demonstrated [40-42]. In this study, we identified 33 TyunGSTs from the T. yunnanensis transcriptome. According to some reports, A Manduca sexta olfactory specific GST called gstmsolf1 is reported as a degradable plant volatile trans-2-hexanal, belonged to the delta subfamily $[40,43]$, in our study, we identified two new GSTs, TyunGST16 and TyunGST29, that were highly expressed in the antennae, and we found that they belonged to the delta/epsilon GST subfamily, Further family classification is needed for the functional speculation of TyunGST16 and TyunGST29. At present, we speculate that they may be related to odor degradation.. TyunGST30 and DaGSTs1 formed a linage in the sigma subfamily. DaGSTs1 may play a role in reducing the negative effects of terpenoids on beetles [44]. We speculate that TyunGST30 also has this function.

Insect CCE is a superfamily, which participates in many physiological processes and contains a variety of substrates [45]. Some of these are secreted enzymes, which refresh ORs by removing redundant esterase odorants from the surrounding area. Besides Z11-16: Ald, Z11-16: Ac is another major sex pheromone component reported in [46]. We found 29 CCE genes in the transcriptome of T. yunnanensis. The total number is higher than that of D. armandi (8) [47] and less than that of T. castaneum (63) [48]. The phylogenetic tree shows that the CCE genes of T. yunnanensis can be divided into five categories. Among them, TyunCCE5 and DarmCCE3 (AYN64425.1) cluster into the same branch, and DarmCCE3 is inferred to play a role in host detoxification [47], which we speculate that TyunCCE5 may also have. Using the FPKM value and a qPCR analysis, we found that three CCEs have a higher expression in the antennae: TyunCCE8, TyunCCE17, and TyunCCE21. TyunCCE8 and DarmCCE2 (AYN64424.1) are clustered into the same clade, whose most enzymes have a dietary detoxification function or ester odor degradation function, such as SICXE10 [49] and SexCXE10 [50] with antennae dominant expression that can degrade ester plant secondary metabolites. Unlike these three CCEs, TyunCCE8, TyunCCE17, and TyunCCE21 have no obvious gender-biased expression. TyunCCE23, DarmCCE (AYN64426.1), and TcasCCE (NP_001180223.1) are divided into the JHE branch. TcasCCE (NP_001180223.1) has been confirmed to have the function of degrading juvenile hormones. We speculate that TyunCCE23 also has this function; of course, this inference needs to be further verified. 


\section{Conclusions}

Our present work has characterized the detoxification gene families of T. yunanensis by transcriptome, together with bioinformatics-based analyses and molecular strategies of qPCR. Our study first lead to the identification of 113 genes associated with detoxification, with as many as 51 CYPs. Further, expression profile studies provided reference data for these genes to explore their potential roles in the detoxification of plant secondary metabolites. Together, this study has complemented the information of detoxification gene families in T. yunanensis and will allow for target experiments to screen potential attractants or repellents and to develop novel pest control strategies for controlling this beetle and protect the balance of the forest ecosystem.

Supplementary Materials: The following supporting information can be downloaded at: https: / / www.mdpi.com/article/10.3390/d14010023/s1, Table S1: The primers designed for qPCR analysis; Table S2: Protein sequence of Tomicus yunnanensis.

Author Contributions: Conceptualization, W.L. and N.Z.; methodology, B.Y. and N.L.; software, J.Z. and Z.L.; validation, W.L., S.Z. and N.Z.; formal analysis, N.L.; investigation, J.Z. and Z.L.; resources, W.L. and B.Y.; data curation, J.Y.; writing—original draft preparation, W.L.; writing-review and editing, W.L. and N.Z.; visualization, N.Z.; supervision, N.Z.; project administration, N.Z.; funding acquisition, N.Z. All authors have read and agreed to the published version of the manuscript.

Funding: The research was supported by the National Natural Science Foundation of China (31760210), the Science Research Foundation of Yunnan Provincial Department of Education Project (2021Y269), and Key Project of Yunnan Applied Basic Research Program (grand No.202101AS070009; 2018FG001-010).

Institutional Review Board Statement: Not applicable.

Data Availability Statement: All Data are provided within the paper and supplementary material.

Conflicts of Interest: All the authors declare that they have no conflict of interest.

\section{References}

1. Kant, M.R.; Jonckheere, W.; Knegt, B.; Lemos, F.; Liu, J.; Schimmel, B.; Villarroel, C.A.; Ataíde, L.; Dermauw, W.; Glas, J.J.; et al. Mechanisms and ecological consequences of plant defence induction and suppression in herbivore communities. Ann. Bot. 2015, 115, 1015-1051. [CrossRef]

2. Richard, K.; Anurag, A.A.; Marc, M. The benefits of induced defenses against herbivores. Ecology 1997, 78, 1351-1355. [CrossRef]

3. Walling, L.L. The Myriad Plant Responses to Herbivores. J. Plant Growth Regul. 2000, 19, 195-216. [CrossRef] [PubMed]

4. Hermsmeier, D.; Schittko, U.; Baldwin, I.T. Molecular interactions between the specialist herbivore Manduca sexta (Lepidoptera.; Sphingidae) and its natural host nicotiana attenuata. I. Large-Scale Changes in the accumulation of growth-and defense-related plant mRNAs. Plant Physiol. 2001, 125, 683-700. [CrossRef] [PubMed]

5. Chen, C.Y.; Kang, Z.J.; Shi, X.Y.; Gao, X.W. Metabolic adaptation mechanisms of insects to plant secondary metabolites and their implications for insecticide resistance of insects. Acta Entomol. Sin. 2015, 58, 1126-1139.

6. Ingelman-Sundberg, M. Genetic variability in susceptibility and response to toxicants. Toxicol. Lett. 2001, 120, 259-268. [CrossRef]

7. Jiang, G.; Zhang, Y.; Chen, F.; Li, J.; Li, X.; Yue, J.; Liu, H.; Li, H.; Ran, C. Differential Analysis of the Cytochrome p450 AcaricideResistance Genes in Panonychus citri (Trombidiformes: Tetranychidae) Strains. Fla. Entomol. 2015, 98, 318-329. [CrossRef]

8. Yi, X.Q.; Di, Y.D.; Zhang, Q. Preliminary Study on Genetic Diversity of Esox reicherti in Amur River Basin Based on Cytochrome b Gene. J. Anhui Agric. Sci. 2009, 37, 5403-5405. [CrossRef]

9. Itokawa, K.; Komagata, O.; Kasai, S.; Kawada, H.; Mwatele, C.; Dida, G.O.; Njenga, S.; Mwandawiro, C.; Tomita, T. Global spread and genetic variants of the two CYP9M10 haplotype forms associated with insecticide resistance in Culex quinquefasciatus Say. Heredity 2013, 111, 216-226. [CrossRef] [PubMed]

10. Schuler, M.A.; Berenbaum, M.R. Structure and Function of Cytochrome P450S in Insect Adaptation to Natural and Synthetic Toxins: Insights Gained from Molecular Modeling. J. Chem. Ecol. 2013, 39, 1232-1245. [CrossRef]

11. Feyereisen, R. Insect CYP Genes and P450 Enzymes 㑔. In Reference Module in Life Sciences; Elsevier: Amsterdam, The Netherlands, 2019. [CrossRef]

12. Mao, Y.B.; Cai, W.J.; Wang, J.W. Silencing a cotton bollworm P450 monooxygenase gene by plant-mediated RNAi impairs larval tolerance of gossypol. Nat. Biotechnol. 2007, 25, 1307-1313. [CrossRef]

13. Liu, S.; Shi, X.-X.; Jiang, Y.-D.; Zhu, Z.-J.; Qian, P.; Zhang, M.-J.; Yu, H.; Zhu, Q.-Z.; Gong, Z.-J.; Zhu, Z.-R. De novo analysis of the Tenebrio molitor (Coleoptera: Tenebrionidae) transcriptome and identification of putative glutathione S-transferase genes. Appl. Entomol. Zool. 2015, 50, 63-71. [CrossRef] 
14. Vontas, J.G.; Small, G.J.; Nikou, D.C.; Ranson, H.; Hemingway, J. Purification, molecular cloning and heterologous expression of a glutathione S-transferase involved in insecticide resistance from the rice brown planthopper, Nilaparvata lugens. Biochem. J. 2002, 362, 329-337. [CrossRef]

15. Friedman, R. Genomic organization of the glutathione S-transferase family in insects. Mol. Phylogenetics Evol. 2011, 61, 924-932. [CrossRef] [PubMed]

16. Ma, J.N.; Dai, L.L.; Zhang, R.R.; Chen, H. Cloning and expression of glutathione S-transferase gene DaGSTe1 of Dendroctonus armandi. J. Northwest A F Univ. 2015, 43, 117-122.

17. Durand, N.; Carot-Sans, G.; Chertemps, T.; Bozzolan, F.; Party, V.; Renou, M.; Debernard, S.; Rosell, G.; Maïbèche-Coisné, M. Characterization of an antenna carboxylesterase from the pest moth Spodoptera littoralis degrading a host Plant Odorant. PLoS ONE 2010, 5, e15026. [CrossRef]

18. Yu, L.F.; Huang, H.G.; Ze, S.Z.; Ren, L.L.; Zong, S.X.; Lu, W.J.; Luo, Y.Q. Research on the spatial distribution patterns of Tomicus sp. in Pinus yunnanensis during the shoot feeding period. Chin. J. Appl. Entomol. 2017, 54, 940-946.

19. Chen, P.; Lu, J.; Haack, R.A.; Ye, H. Attack pattern and reproductive ecology of Tomicus brevipilosus (Coleoptera: Curculionidae) on Pinus yunnanensis in Southwestern China. J. Insect Sci. 2015, 15, 43. [CrossRef] [PubMed]

20. Wang, X.W.; Chen, P.; Wang, Y.X.; Yuan, R.L.; Feng, D.; Li, L.S.; Ye, H.; Pan, Y.; Lv, J.; Zhou, Y.F.; et al. Population Structure and Succession Law of Tomicus Species in Yunnan. For. Res. 2018, 31, 167-172.

21. Lü, J.; Hu, S.J.; Ma, X.Y.; Chen, J.M.; Li, Q.Q.; Ye, H.; Arthofer, W. Origin and Expansion of the Yunnan Shoot Borer, Tomicus yunnanensis (Coleoptera: Scolytinae): A Mixture of Historical Natural Expansion and Contemporary Human-Mediated Relocation. PLoS ONE 2014, 9, e111940. [CrossRef]

22. Wang, J.; Zhang, Z.; Kong, X.; Wang, H.; Zhang, S. Intraspecific and interspecific attraction of three Tomicus beetle species during the shoot-feeding phase. Bull. Entomol. Res. 2015, 105, 225-233. [CrossRef]

23. Grabherr, M.G.; Haas, B.J.; Yassour, M.; Levin, J.Z.; Thompson, D.A.; Amit, I.; Adiconis, X.; Fan, L.; Raychowdhury, R.; Zeng, Q.; et al. Full-length transcriptome assembly from RNA-Seq data without a reference genome. Nat. Biotechnol. 2011, 29, 644-652. [CrossRef] [PubMed]

24. Li, B.; Dewey, C. RSEM: Accurate transcript quantification from RNA-Seq data with or without a reference genome. BMC Bioinform. 2011, 12, 323. [CrossRef] [PubMed]

25. Cock, P.J.A.; Christopher, J.F.; Naohisa, G.; Michael, L.H.; Peter, M.R. The Sanger FASTQ file format for sequences with quality scores, and the Solexa/Illumina FASTQ variants. Nucleic Acids Res. 2010, 38, 1767-1771. [CrossRef] [PubMed]

26. Trapnell, C.; Williams, B.A.; Pertea, G.; Mortazavi, A.; Kwan, G.; Van Baren, M.J.; Salzberg, S.L.; Wold, B.J.; Pachter, L. Transcript assembly and quantification by RNA-Seq reveals unannotated transcripts and isoform switching during cell differentiation. Nat. Biotechnol. 2010, 28, 511-515. [CrossRef] [PubMed]

27. Feyereisen, R. 8-Insect CYP Genes and P450 Enzymes. In Insect Molecular Biology and Biochemistry; Academic Press: San Diego, CA, USA, 2012; pp. 236-316. [CrossRef]

28. Kumar, S.; Stecher, G.; Tamura, K. MEGA7: Molecular Evolutionary Genetics Analysis version 7.0 for bigger datasets. Mol. Biol. Evol. 2016, 33, 1870-1874. [CrossRef]

29. Balakrishnan, S.; Gao, S.; Lercher, M.J.; Hu, S.; Chen, W.H. Evolview v3: A webserver for visualization, annotation, and management of phylogenetic trees. Nucleic Acids Res. 2019, 47, W270-W275. [CrossRef]

30. Singh, V.K.; Mangalam, A.K.; Dwivedi, S.; Naik, S. Primer Premier: Program for Design of Degenerate Primers from a Protein Sequence. BioTechniques 1998, 24, 318-319. [CrossRef]

31. Simon, P. Q-Gene: Processing quantitative real-time RT-PCR data. Bioinformatics 2003, 19, 1439-1440. [CrossRef]

32. Shi, H.; Pei, L.; Gu, S.; Zhu, S.; Wang, Y.; Zhang, Y.; Li, B. Glutathione S-transferase (GST) genes in the red flour beetle, Tribolium castaneum, and comparative analysis with five additional insects. Genomics 2012, 100, 327-335. [CrossRef]

33. Schama, R.; Pedrini, N.; Juárez, M.P.; Nelson, D.R.; Torres, A.Q.; Valle, D.; Mesquita, R.D. Rhodnius prolixus supergene families of enzymes potentially associated with insecticide resistance. Insect. Biochem. Mol. Biol. 2016, 69, 91-104. [CrossRef]

34. Feyereisen, R. Evolution of insect P450. Biochem. Soc. Trans. 2006, 34, 1252-1255. [CrossRef]

35. Liu, N.Y.; Li, Z.B.; Zhao, N.; Song, Q.S.; Zhu, J.Y.; Yang, B. Identification and characterization of chemosensory gene families in the bark beetle, Tomicus yunnanensis. Comp. Biochem. Physiol. Part D Genom. Proteom. 2018, 25, 73-85. [CrossRef]

36. Liu, N.N.; Li, M.; Gong, Y.H.; Liu, F.; Li, T. Cytochrome P450s-Their expression, regulation, and role in insecticide resistance. Pestic. Biochem. Physiol. 2015, 120,77-81. [CrossRef]

37. Bozzolan, F.; Siaussat, D.; Maria, A.; Durand, N.; Pottier, M.-A.; Chertemps, T.; Maïbèche-Coisne, M. Antennal uridine diphosphate (UDP)-glycosyltransferases in a pest insect: Diversity and putative function in odorant and xenobiotics clearance. Insect. Mol. Biol. 2014, 23, 539-549. [CrossRef]

38. Claudia, C.R.; María, F.L.; Ana, K.C.A.; Verónica, P.M.; Brian, T.S.; Gerardo, Z. Isolation and expression of cytochrome P450 genes in the antennae and gut of pine beetle Dendroctonus rhizophagus (Curculionidae: Scolytinae) following exposure to host monoterpenes. Gene 2013, 520, 47-63. [CrossRef]

39. Pottier, M.A.; Bozzolan, F.; Chertemps, T.; Jacquin-Joly, E.; Lalouette, L.; Siaussat, D.; Maibèche-Coisne, M. Cytochrome P450s and cytochrome P450 reductase in the olfactory organ of the cotton leafworm Spodoptera littoralis. Insect Mol. Biol. 2012, 21, 568-580. [CrossRef] 
40. He, P.; Zhang, Y.-F.; Hong, D.-Y.; Wang, J.; Wang, X.-L.; Zuo, L.-H.; Tang, X.-F.; Xu, W.-M.; He, M. A reference gene set for sex pheromone biosynthesis and degradation genes from the diamondback moth, Plutella xylostella, based on genome and transcriptome digital gene expression analyses. BMC Genom. 2017, 18, 219. [CrossRef]

41. You, Y.; Xie, M.; Ren, N.; Cheng, X.; Li, J.; Ma, X.; Zou, M.; Vasseur, L.; Gurr, G.M.; You, M. Characterization and expression profiling of glutathione S-transferasesin the diamondback moth, Plutella xylostella (L.). BMC Genom. 2015, 16, 152. [CrossRef]

42. Zou, X.P.; Xu, Z.B.; Zou, H.W.; Liu, J.S.; Chen, S.N.; Feng, Q.L.; Zheng, S.C. Glutathione S-transferase SIGSTE1 in Spodoptera litura may be associated with feeding adaptation of host plants. Insect. Biochem. Mol. Biol. 2016, 70, 32-43. [CrossRef]

43. Rogers, M.E.; Jani, M.K.; Vogt, R.G. An olfactory-specific glutathione-S-transferase in the sphinx moth Manduca sexta. J. Exp. Biol. 1999, 202, 1625-1637. [CrossRef] [PubMed]

44. Dai, L.L.; Ma, J.N.; Ma, M.Y.; Zhang, H.Q.; Shi, Q.; Zhang, R.; Chen, H. Characterisation of GST genes from the Chinese white pine beetle Dendroctonus armandi (Curculionidae: Scolytinae) and their response to host chemical defence. Pest Manag. Sci. 2016, 72, 816-827. [CrossRef] [PubMed]

45. Oakeshott, J.G.; Claudianos, C.; Campbell, P.M.; Newcomb, R.D.; Russell, R.J. Biochemical Genetics and Genomics of Insect Esterases. Sci. Ref. Modul. Life Sci. 2019, 5, 309-381. [CrossRef]

46. Tamaki, Y.; Kawasaki, K.; Yamada, H.; Koshihara, T.; Osaki, N.; Ando, T.; Yoshida, S.; Kakinohana, H. Z-11-hexadecenal and Z-11-hexadecenyl acteact: Sex pheromone components of the Diamonback moth (Lepideptera: Plutellidae). Appl. Entomol. Zool. 1997, 12, 208-210. [CrossRef]

47. Dai, L.L.; Gao, H.M.; Ye, J.Q.; Fu, D.Y.; Sun, Y.Y.; Chen, H. Isolation of CarE genes from the Chinese white pine beetle Dendroctonus armandi (Curculionidae: Scolytinae) and their response to host chemical defense. Pest Manag. Sci. 2019, 75, 986-997. [CrossRef] [PubMed]

48. Zhao, Y.J.; Wang, Z.Q.; Zhu, J.Y.; Liu, N.Y. Identification and characterization of detoxification genes in two cerambycid beetles, Rhaphuma horsfieldi and Xylotrechus quadripes (Coleoptera: Cerambycidae: Clytini). Comp. Biochem. Physiol. Part B Biochem. Mol. Biol. 2020, 243-244, 110431. [CrossRef]

49. Durand, N.; Carot-Sans, G.; Chertemps, T.; Montagné, N.; Jacquin-Joly, E.; Debernard, S.; Maïbèche-Coisne, M. A diversity of putative carboxylesterases are expressed in the antennae of the noctuid moth Spodoptera littoralis. Insect Mol. Biol. 2010, 19, 87-97. [CrossRef] [PubMed]

50. He, P.; Zhang, Y.N.; Yang, K.; Li, Z.Q.; Dong, S.L. An antenna-biased carboxylesterase is specifically active to plant volatiles in Spodoptera exigua. Pestic. Biochem. Physiol. 2015, 123, 93-100. [CrossRef] 